\title{
Cohomology of groups and almost periodic extensions of minimal sets
}

\author{
ROBERT ELLIS † \\ From the University of Minnesota, USA
}

(Received 26 September 1980)

\begin{abstract}
Let $\mathscr{B}$ and $\mathscr{C}$ be minimal flows which are disjoint over their intersection. A dynamical interpretation is given of the groups $H^{1}(B / S, C / S)$ and $H^{2}(B / S, C / S)$, where $B$ and $C$ are the groups of $\mathscr{B}$ and $\mathscr{C}$ respectively, and $S$ is the group of the flow $\mathscr{B} \vee \mathscr{C}$.
\end{abstract}

\section{Introduction}

Let $(K, X, T)$ be a bitransformation group where $(X, T)$ is a minimal flow with compact phase space $X$ and phase group $T$, and where $K$ is a compact topological group. Then the flows $(Z, T)$ 'between' $(X, T)$ and $(X / K, T)$ are completely determined by the closed subgroups of $K$, and so a classification of the latter produces one of the former. This provides the basis for the introduction of the cohomology of groups into topological dynamics.

For example, let $J$ be a closed normal subgroup of $K, J^{\perp}$ the set of closed subgroups $S$ of $K$ with $K=J \cdot S$ (semidirect product) and $L \in J^{\perp}$. Then it is known that $J^{\perp}$ is in one-one correspondence with $Z^{1}(L, J)$ and $H^{1}(L, J)$ represents $J^{\perp} / R$ where $L_{1} \equiv L_{2}(\bmod R)$ if $L_{2}=a L_{1} a^{-1}$ for some $a \in J$.

The dynamical interpretation of the preceding statement is best given in terms of algebras (or equivalently pointed flows) where the connection between flow and subgroups is precise (see [1]).

Thus let $\mathscr{S}$ be an almost periodic extension of $\mathscr{A}$ with $S$ normal in $A, C$ a $\tau$-closed normal subgroup of $A$ with $S \subset C, C^{\perp}$ the set of closed normal subgroups $N$ of $A$ with $C N=A$ and $C \cap N=S$, and $B \in C^{\perp}$. (Here $A$ and $S$ are the groups of $\mathscr{A}$ and $\mathscr{S}$ respectively.) If $|\mathscr{S}| \cong(X, T)$ and $|\mathscr{A}| \cong(X / K, T)$, then $A / S \cong K$ and we may take $C / S \cong J$ and $B / S \cong L$. Now let $\mathscr{C}^{\perp}$ be the set of $T$-subalgebras $\mathcal{N}$ of $\mathscr{S}$ such that $\mathscr{C} \vee \mathcal{N}=\mathscr{S}$ and $\mathscr{C} \cap \mathcal{N}=\mathscr{A}$. Then $\mathscr{C}^{\perp}$ is in one-one correspondence with $C^{\perp}$. Thus $H^{1}(B / S, C / S)$ classifies $\mathscr{C}^{\perp} / R$, and $H^{1}(B / S, C / S), \mathscr{C}^{\perp} / R$ where $\mathscr{B}_{1} \equiv \mathscr{B}_{2}(R)$ if $\mathscr{B}_{2}=\mathscr{B}_{1} \alpha$ for some $\alpha \in C$.

The dynamical interpretation becomes even more transparent when there exists a cocycle $\sigma$ on $\mathscr{A}$ to $A / S$ such that $\mathscr{C}=\operatorname{ext}(\mathscr{A}, \sigma)$ and $\mathscr{B}=\operatorname{per}(\mathscr{S}, \sigma)$. In this case the various elements of $\mathscr{C}^{\perp}$ may be obtained from $\mathscr{B}$ by perturbing it by the set of $\sigma$-cocycles on $\mathscr{A}$ to $C / S$ (see 3.12).

† Address for correspondence: Dr Robert Ellis, Department of Mathematics, University of Minnesota, Minneapolis, MN 55455, USA. 
After considering $H^{1}(L, J)$ it is natural to enquire into $H^{2}(L, J)$. In order to apply the standard theory one must assume that $J$ is abelian. Then a 2-cocycle $f$ on $L$ to $J$ induces a new group structure $(K, f)$ on $K$. (For the sake of simplicity I avoid topological considerations at this point.)

There are now two problems: (1) define an action of $(K, f)$ on $X$; and (2) define a new action of $T$ on $X$ which commutes with the new action of $K$.

It turns out that there is a natural way to solve (1) (5.3) and that in many cases (for example when $T=\mathbb{Z}$ ) (2) may be accomplished by perturbing the original flow on $X$.

In discussing the dynamical interpretation of $H^{1}$ the algebraic point of view is convenient but not essential. However, the situation is different with respect to $H^{2}$. The reason is that the link between the new group structure $(K, f)$ and the new flow produced in answer to (2) is provided by the group of the latter. Reverting to the 'algebraic' terminology introduced above and denoting this flow by $\mathcal{N}$ and its group by $N$, it turns out that there is an injective map $\Gamma$ from $A / N$ into $A / S(=K)$ such that the new group structure $(A / S, f)$ is obtained by carrying over the group structure on $A / N$ by means of $\Gamma$.

These new flows are of the form per $(\mathscr{S}, \rho)$ where $\rho \in Z_{B}(\mathscr{C}, C / S)$, the set of $B$-cocycles on $\mathscr{C}$ to $C / S$ (see 5.4). Moreover, there is a homomorphism $\Delta: Z_{B}(\mathscr{C}, C / S) \rightarrow Z^{2}(B / S, C / S)$ which induces as isomorphism of $Z_{B}(\mathscr{C}, C / S) / Z_{B}(\mathscr{P})$ onto $H^{2}(B / S, C / S)$ when $T=\mathbb{Z}$. (Here $Z_{B}(\mathscr{S})$ is the subset of $Z_{B}(\mathscr{C}, C / S)$ consisting of those $\rho$ for which per $(\mathscr{S}, \rho)=\mathscr{S}$, i.e. the perturbed flow coincides with the original one - see (5.20).)

\section{The cohomology $H(L, J)$}

In this section the purely algebraic aspects of the situation will be discussed. The material is well known in the context of group theory and I include it for the sake of completeness. Since the proofs are standard I shall omit them. The interested reader may consult [5] or supply them himself since they are quite straightforward. A word of caution, however: the groups considered in this section come provided with a topology so that the subgroups involved are closed and the cocycles considered are continuous.

(2.1) Standing notation. Henceforth $K$ will denote a compact topological group, $J$ a closed normal subgroup of $K$ and $L$ a fixed element of $J^{\perp}$, the set of closed subgroups $S$ of $K$ such that $K$ is the semidirect product of $J$ and $S$. Thus $K=J+S$ and $J \cap S=\{0\}$. (It is convenient to write the group operation in $K$ additively although $K$ need not be abelian.)

(2.2) Definition. A cocycle on $L$ to $J$ is a continuous function $z: L \rightarrow J$ such that $z(0)=0$ and $z(a+b)=z(a)+a \cdot z(b)(a, b \in L)$. (Here $a \cdot x=a+x-a \quad(a \in L$, $x \in J)$.) The set of cocycles on $L$ to $J$ will be denoted by $Z(L, J)$ or $Z$.

(2.3) Definition. A coboundary on $L$ to $J$ is a continuous function $z: L \rightarrow J$ such that $z(0)=0$ and $z(a)=c \cdot a-a$ for some $c \in J$ and all $a \in L$. The set of coboundaries on $L$ to $J$ will be denoted by $B(L, J)$ or $B$. If $c \in J$ then $z_{c}$ will denote the coboundary 
$a \rightarrow c \cdot a-a: L \rightarrow J$. (Note that $z(a)=c \cdot a-a=c+a-c-a \in J$ since $J$ is normal in $K$.)

Let $S \in J^{\perp}$ and $a \in K$. Then $a S$ and $a J$ will denote the unique elements of $S$ and $J$ respectively such that $a=a J+a S$. (Note that $a J$ depends upon $a S$. Thus the notation is imprecise.)

(2.4) Proposition. Given $z: L \rightarrow J$ let $S_{z}=\{a \in K \mid z(a L)=a J\}$ and given $S \in J^{\perp}$ let $z_{S}: L \rightarrow J$ be such that $z_{S}(x)+x \in S(x \in L)$. Then

(1) $S_{z} \in J^{\perp}(z \in Z(L, J))$.

(2) $z_{S} \in Z(L, J)\left(S \in J^{\perp}\right)$.

(3) The map $z \in S_{z}: Z(L, J) \rightarrow J^{\perp}$ is bijective with inverse $S \rightarrow z_{S}: J^{\perp} \rightarrow Z(L, J)$.

(2.5) Definition. The action of $J$ on $Z(L, J)$. Let $c \in J$ and $z \in Z(L, J)$. Then $c z$ is the $\operatorname{map} a \rightarrow c+z(a)-a \cdot c: L \rightarrow J$.

Notice that when $J$ is abelian $\left(z_{c}+z\right)(a)=c \cdot a-a+z(a)=c+a-c-a+z(a)=$ $c-a \cdot c+z(a)=c+z(a)-a \cdot c=c z(a)(a \in J)$. Thus $c z=z_{c}+z(c \in J)$ when $J$ is abelian.

(2.6) Proposition. (1) $B \subset Z$.

(2) $c z \in Z(c \in J, z \in Z)$.

(3) The map $(c, z) \mapsto c z: J \times Z \rightarrow Z$ defines an action of $J$ on $Z$.

(4) Let $c, d \in J$, then $z_{c}=z_{d}$ if and only if $c z=d z(z \in Z)$.

Thus $B$ acts on $Z$ and we may form $H=Z / B=\{B+z / z \in Z\}$. When $J$ is abelian $Z$ and $B$ are groups and $H$ is the quotient group.

(2.7) LemMA. Let $f_{z}$ be the map $a \rightarrow z(a L)+a L: K \rightarrow K(z \in Z)$. Then

(1) $f_{z}$ is a homomorphism of $K$ into $K$.

(2) $S_{z}=\left\{a \mid f_{z}(a)=a\right\}$.

(3) $f_{d z}=d \cdot f_{z}(d \in J)$.

(4) $S_{d z}=d \cdot S_{z}\left(=d+S_{z}-d\right)(d \in J)$.

(2.8) Proposition. Set $S_{1} \equiv S_{2}(R)$ if $S_{2}=c \cdot S_{1}=c+S_{1}-c$ for some $c \in J$ $\left(S_{1}, S_{2} \in J^{\perp}\right)$. Then

(1) $S_{z} \equiv S_{x}$ if and only if $z=c x$ for some $c \in J(z, x \in Z)$.

(2) The map $z \rightarrow S_{z}: Z \rightarrow J^{\perp}$ induces a one-one correspondence between $H(L, J)$ and $J^{\perp} / R$.

3. The dynamical interpretation

Here the results of $\S 2$ will be used to discuss a situation which occurs frequently in topological dynamics.

(3.1) The data. $\mathscr{A}$ and $\mathscr{S}$ will denote minimal flows such that $\mathscr{P}$ is an almost periodic extension of $\mathscr{A}$ and $S$ is normal in $A$. (Here $A$ and $S$ are the groups of $\mathscr{A}$ and $\mathscr{S}$ respectively.)

$C$ and $B$ will denote $\tau$-closed subgroups of $A$ such that $C$ is normal, $C B=A$ and $C \cap B=S$.

If one sets $(X, T)=(|\mathscr{S}|, T), K=A / S, J=C / S$ and $L=B / S$, then one obtains the situation described in the introduction. The flow $(|\mathscr{A}|, T)$ is just $(X / K, T)$. 
(3.2) Proposition. Let $\mathscr{C}=\mathfrak{a}(C) \cap \mathscr{S}$ and $\mathscr{B}=\mathfrak{a}(B) \cap \mathscr{S}$. Then $\mathscr{B}$ and $\mathscr{C}$ are disjoint over their intersection, $\mathscr{A}$ and $\mathscr{B} \vee \mathscr{C}=\mathscr{S}$.

Proof. The fact that $\mathscr{B} \cap \mathscr{C}=\mathscr{A}$ and $\mathscr{B} \vee \mathscr{C}=\mathscr{S}$ follows immediately from the Galois theory of distal extension [1, chapter 13].

Since $C=\mathbf{g}(\mathscr{C})$ is normal in $A, \mathscr{C} \beta=\mathscr{C}(\beta \in B=\mathbf{g}(\mathscr{B}))$. Hence $\mathscr{C}$ and $\mathscr{B}$ are disjoint over their intersection $[1,18.9]$.

(3.3) Proposition. Let $\mathscr{C}^{\perp}$ be the set of T-subalgebras $\mathscr{L}$ of $\mathscr{S}$ with $\mathscr{C} \vee \mathscr{L}=\mathscr{S}$, $\mathscr{C} \cap \mathscr{L}=\mathscr{A}$ and $C^{\perp}$ the set of $\tau$-closed subgroups $L$ of $A$ with $C L=A, C \cap L=S$. Then the map $\psi: C^{\perp} \rightarrow \mathscr{C}^{\perp}$ such that $\psi(L)=\mathrm{a}(L) \cap \mathscr{S}$ is a bijection and $c L_{2} c^{-1}=L_{1}$ if and only if $\psi\left(L_{1}\right) c=\psi\left(L_{2}\right)\left(L_{1}, L_{2} \in C^{\perp}, c \in C\right)$.

Proof. This follows immediately from the results of chapter 13 of [1].

(3.4) Remarks. Proposition 3.3 and the results of $\S 2$ establish a one-one correspondence between $Z(B / S, C / S)$ and $\mathscr{C}^{\perp}$, and one between $H(B / S, C / S)$ and $\mathscr{C}^{\perp} / R$ where $\mathscr{L}_{1} \equiv \mathscr{L}_{2}(\bmod R)$ if $\mathscr{L}_{2}=\mathscr{L}_{1} c$ for some $c \in C$. In terms of flows $\mathscr{L}_{1} \equiv \mathscr{L}_{2}(\bmod R)$ if and only if there exists a flow isomorphism $\psi:\left|\mathscr{L}_{1}\right| \rightarrow\left|\mathscr{L}_{2}\right|$ such that figure 1 is commutative where $\pi_{1}$ and $\pi_{2}$ are the canonical maps.

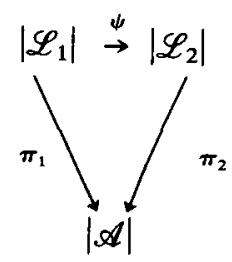

FIGURE 1

In order to obtain some more information about the class $\mathscr{C}^{\perp} \mathrm{I}$ assume that there exists a cocycle $\sigma$ on $\mathscr{A}$ to $A / S$ such that $\mathscr{C}=\operatorname{ext}(\mathscr{A}, \sigma)$ and $\mathscr{B}=\operatorname{per}(\mathscr{Y}, \sigma)$. Such a cocycle will exist when the map $[\rho] \rightarrow \bar{f}_{\rho}: H(\mathscr{A}, A / S) \rightarrow \operatorname{Hom}\left(A / A^{*}, A / S\right)$ is onto and this condition will hold when $|\mathscr{A}|$ is 0 -dimensional. (For a more complete discussion see [2], in particular 3.2, 3.7 and 5.22.) When this situation obtains, the groups of the various flows are given by: $C=\operatorname{ker} \delta \sigma \mid A$, and $B=\{a \in A: \delta \sigma(a)=S a\}$. (Here $\delta \sigma=f_{\sigma}$ in [2] and, consequently, $\delta \sigma(a x)=\delta \sigma(a)+\delta \sigma(x)(a \in A, x \in \beta T)$.)

(3.5) Lemma. Let $\beta(x, t)=\pi(\sigma(x, t))(x \in|\mathscr{A}|, t \in T)$ where $\pi: A / S \rightarrow B / S$ is the canonical homomorphism. Then $\beta$ is cohomologous to $\sigma(\bmod \mathscr{A})$.

Proof. Let $\delta \sigma$ and $\delta \beta$ be the functions corresponding to $\sigma$ and $\beta$ respectively (see [2] and [3]). Then $\delta \beta(a)=\pi(\delta \sigma(a))(a \in A)$.

Let $a=c b$ with $c \in C$ and $b \in B$. Then $\delta \sigma(a)=\delta \sigma(c)+\delta \sigma(b)=\delta \sigma(b)=\pi \delta \sigma(a)=$ $\delta \beta(a)$ since $c \in \operatorname{ker} \delta \sigma$. The proof is completed.

Lemma 3.5 implies that $\mathscr{C}=\operatorname{ext}(\mathscr{A}, \beta)$ and $\mathscr{B}=\operatorname{per}(\mathscr{Y}, \beta)$. Hence we may assume that $\sigma$ is a cocycle on $\mathscr{A}$ to $B / S$.

The cocycle $\sigma$ produces a new flow $(x, t) \rightarrow x \circ t=\sigma(x \mid \mathscr{A}, t)^{-1} x t:|\mathscr{S}| \times T \rightarrow|\mathscr{S}|$ and $|\mathscr{B}|$ may be identified with the subset $\left\{x_{0} \circ p \mid p \in M\right\}$; where $x_{0}=u \mid \mathscr{S}$ is the base point 
of $(|\mathscr{S}|, T)$. Here $M$ is a fixed minimal subset of the flow $(\beta T, T)$ (see [1]). In order to avoid confusion I shall denote the set $\left\{x_{0} \circ p \mid p \in M\right\}$ by $W$.

The group $A / S$ acts on $|\mathscr{S}|$ via the map $(a S, p \mid \mathscr{S}) \rightarrow a p|\mathscr{S}: A / S \times| \mathscr{T}|\rightarrow| \mathscr{S} \mid$. I shall denote $a S$ by $\bar{a}$ and $\bar{a} x$ by $a x(a \in A, x \in|\mathscr{S}|)$ when there is no chance of confusion. Also $x \mid \mathscr{A}$ will be denoted by $\bar{x}(x \in|\mathscr{P}|)$.

(3.6) Proposition. (1) $a x \circ t=\left(\sigma(\bar{x}, t)^{-1} \cdot a\right)(x \circ t)(x \in|\mathscr{P}|, a \in A, t \in T)$. (Recall that $\sigma(\bar{x}, t) \cdot a=\sigma(\bar{x}, t)+\bar{a}-\sigma(\bar{x}, t)$.)

(2) $a(x \circ t)=((\sigma(\bar{x}, t) \cdot a) x) \circ t \quad(x \in|\mathscr{P}|, a \in A, t \in T)$.

(3) $x_{0} \circ c=x_{0} c \quad(c \in C)$.

(4) $c x_{0}{ }^{\circ} q=x_{0}{ }^{\circ} c q \quad(c \in C, q \in \beta T)$.

(5) $c x \in W \quad(c \in C, x \in W)$.

(6) $x_{1}\left|\mathscr{A}=x_{2}\right| \mathscr{A}$ if and only if $x_{2}=c x_{1}$ for some $c \in C \quad\left(x_{1}, x_{2} \in|\mathscr{B}|\right)$.

(7) $W=\{x \in|\mathscr{S}|: \delta \sigma(x)=0\}$.

Proof. (1) ax॰ $t=\sigma(\bar{x}, t)^{-1} a x t=\sigma(\bar{x}, t)^{-1} a \sigma(\bar{x}, t) \sigma(\bar{x}, t)^{-1} x t=\left(\sigma(\bar{x}, t)^{-1} \cdot a\right)(x \circ t)$.

(2) Replace $a$ by $\sigma(\tilde{x}, t) \cdot a$ in (1).

(3) $x_{0} \circ c=\sigma\left(\bar{x}_{0}, c\right)^{-1} x_{0} c=\delta \sigma(c)^{-1} x_{0} c=x_{0} c$. (Recall that $C=\operatorname{ker} \delta \sigma$.)

(4) Since $c x_{0}=x_{0} c, x_{0} \circ c q=\left(x_{0} \circ c\right) \circ q=c x_{0} \circ q$.

(5) Let $x \in W$. Then $x=x_{0} \circ q$ for some $q \in M$. Hence $c x=c\left(x_{0} \circ q\right)=$ $\left(\left(\sigma\left(\bar{x}_{0}, q\right) \cdot c\right) x_{0}\right) \circ q$ by $(2)$. Since $\sigma\left(\bar{x}_{0}, q\right) \cdot c=S d$ for some $d \in C, c x=d x_{0}^{\circ} q=$ $x_{0} \circ d q \in W$ by (4).

(6) Let $x_{1}=x_{0} \circ p$ and $x_{2}=x_{0} \circ q$ for some $p, q \in M$ and let $x_{1}\left|\mathscr{A}=x_{2}\right| \mathscr{A}$. Then $q|\mathscr{S}=a p| \mathscr{S}$ for some $a \in A$. Let $a=b c$ with $c \in C$ and $b \in B$. Then $x_{2}=x_{0}{ }^{\circ} q=$ $x_{0} \circ b c p=x_{0} \circ c p$ (since $\left.x_{0} \circ b=x_{0}\right)$. Furthermore, $x_{0} \circ c p=\left(x_{0} \circ c\right) \circ p=x_{0} c \circ p$ by (3); and $x_{0} c \circ p=\left(\delta \sigma(p)^{-1} \cdot c\right)\left(x_{0} \circ p\right)$ by (2). Hence $x_{2}=\left(\delta \sigma(p)^{-1} \cdot c\right) \quad\left(x_{0} \circ p\right)=$ $\left(\delta \sigma(p)^{-1} \cdot c\right) x_{1}$ and $\delta \sigma(p)^{-1} \cdot c \in C / S$.

Clearly $x_{2}=c x_{1}$ for some $c \in C$ implies that $x_{2}\left|\mathscr{A}=x_{1}\right| \mathscr{A}$.

(7) Let $x \in W$. Then $x=x_{0}{ }^{\circ} p=\delta \sigma(p)^{-1} x_{0} p=x_{0} q$ for some $p \in M$, where $q=$ $b^{-1} p, \delta \sigma(p)=S b$. Hence $x=q \mid \mathscr{S}$ and $\delta \sigma(x)=\delta \sigma(q)=\delta \sigma\left(b^{-1} p\right)=\delta \sigma\left(b^{-1}\right) \delta \sigma(p)=$ $S b^{-1} \mathrm{~S} b=0$.

Now let $x \in|\mathscr{S}|$ with $\delta \sigma(x)=0$; i.e. $x=p \mid \mathscr{S}$ and $\delta \sigma(p)=0$. Then $x=p \mid \mathscr{S}=x_{0} p=$ $\delta \sigma(p)^{-1} x_{0} p=x_{0}{ }^{\circ} p \in W$.

(3.7) Remarks. (1) Statements (5) and (6) shows that $C / S$ acts on $W$ and that $W / C$ is $=|\mathscr{A}|$. However as (1) shows, $(C / S, W, T)$ is in general not a bitransformation group. In their paper [4] Keynes and Newton consider a similar situation. There $\sigma$ is a constant cocycle and what corresponds to $(W, T)$ is called a $\sigma$-extension of $(|\mathscr{A}|, T)$.

(2) The transformation group $(A / S,|\mathscr{P}|)$ may be viewed as a principal bundle over $|\mathscr{A}|$. Then (5) and (6) show that $W$ provides a reduction of the group of this bundle to $C / S$. There is then a cross-section of the canonical map from $|\mathscr{S}| / C / S \rightarrow|\mathscr{A}|$. By examining the groups of the flows involved, one sees that $|\mathscr{S}| / C / S=|\mathscr{C}|$. Moreover, since $C \triangleleft A, \mathscr{C} b=\mathscr{C}(b \in B)$. Consequently, $B / S$ acts on $|\mathscr{C}|$ and $|\mathscr{A}|=|\mathscr{C}| / B / S$. The existence of a cross-section of the map $|\mathscr{C}| \rightarrow|\mathscr{A}|$ implies that topologically $|\mathscr{C}|=$ $B / S \times|\mathscr{A}|$. The cross-section also induces a cocycle on $\mathscr{A}$ to $B / S$ (see [2]) which a straightforward computation reveals to be $\sigma$. 
(3.8) Definition. A $\sigma$-cocycle on $\mathscr{A}$ to $C / S$ is a continuous function $\rho:|\mathscr{A}| \times T \rightarrow C / S$ such that $\rho(x, t s)=\rho(x, t)+\sigma(x, t) \cdot \rho(x t, s)(x \in|\mathscr{A}|, t, s \in T)$. The set of $\sigma$-cocycles on $\mathscr{A}$ to $C / S$ will be denoted by $Z_{\sigma}(\mathscr{A}, C / S)$. (Written multiplicatively, $\rho(x, t s)=$ $\rho(x, t) \sigma(x, t) \rho(x t, s) \sigma(x, t)^{-1}$.)

One may use $\sigma$ to define an action of $T$ on $\mathscr{C}(|\mathscr{A}|, C / S)$, viz. $(t \cdot f)(x)=$ $\sigma(x, t) \cdot f(x t)(x \in|\mathscr{A}|, t \in T)$. Then the definition given above coincides with the usual one in the cohomology of groups (see, for example, [5]).

A $\sigma$-coboundary on $\mathscr{A}$ to $C / S$ is then a cocycle $\gamma$ of the form, $\gamma(x, t)=$ $f(x)-\sigma(x, t) \cdot f(x t)$ for some continuous function $f$ on $|\mathscr{A}|$ to $C / S$. The set of $\sigma$-coboundaries is denoted by $B_{\sigma}(\mathscr{A}, C / S)$.

The reason for the introduction of $\sigma$-cocycles is that they may be used to perturb the flow $(W, T)$. Thus let $\rho \in Z_{\sigma}(\mathscr{A}, C / S)$ and $x \cdot t=\left(\rho(\bar{x}, t)^{-1} x\right) t(x \in W, t \in T)$. Then it is easy to verify that the map $(x, t) \rightarrow x \cdot t: W \times T \rightarrow W$ defines an action of $T$ on $W$. The resulting flow will be denoted by $\left(W_{\rho}, T\right)$.

A computation similar to the one involved in (1) of (3.6) shows that

$$
(a x) \cdot t=\left(\left(\sigma(\bar{x}, t) \rho(\bar{x}, t)^{-1}\right) \cdot a\right)(x \cdot t) \quad(x \in W, a \in C, t \in T) .
$$

(3.9) Proposition. Let $\rho \in Z_{\sigma}(\mathscr{A}, C / S)$. Then $\left(W_{\rho}, T\right)$ is an almost periodic extension of $(|\mathscr{A}|, T)$.

Proof. Let $\alpha$ be an index on $W$. We must find an index $\beta$ on $W$ such that if $(x, y) \in \beta$ with $x|\mathscr{A}=y| \mathscr{A}$ then $(x \cdot t, y \cdot t) \in \alpha(t \in T)$.

If this were impossible, there would exist a net of indices $\beta_{n}$ converging to the diagonal $\Delta$, a net $\left(x_{n}, y_{n}\right)$ with $x_{n}\left|\mathscr{A}=y_{n}\right| \mathscr{A}$ and $\left(x_{n}, y_{n}\right) \in \beta_{n}$ for all $n$ and a net $t_{n}$ with $\left(x_{n} \cdot t_{n}, y_{n} \cdot t_{n}\right) \notin \alpha$ for all $n$.

Let $y_{n}=\bar{c}_{n} x_{n}$ with $c_{n} \in C$. Then, by the compactness of the various spaces involved, we may assume that $\lim x_{n}=z=\lim y_{n}, \bar{c}_{n} \rightarrow \bar{c}, \sigma\left(\bar{x}_{n}, t\right) \rightarrow \bar{b}, \rho\left(\bar{x}_{n}, t_{n}\right)^{-1} \rightarrow \bar{d}$, and $x_{n} \cdot t_{n} \rightarrow w$.

Since $\quad z=\lim y_{n}=\lim \bar{c}_{n} x_{n}=\bar{c} \lim x_{n}=\bar{c} z, \quad \bar{c}=\bar{e} . \quad$ Hence $\lim y_{n} \cdot t_{n}=$ $\lim \left(\bar{c}_{n} x_{n}\right) \cdot t_{n}=\lim \left(\sigma\left(\bar{x}_{n}, t_{n}\right) \rho\left(\bar{x}_{n}, t_{n}\right)^{-1} \cdot \bar{c}_{n}\right)\left(x_{n} \cdot t_{n}\right)=(\overline{b d} \cdot \bar{e})(w)=w, \quad$ a $\quad$ contradiction.

(3.10) Definition. Let $\rho \in Z_{\sigma}(\mathscr{A}, C / S)$. Then $\operatorname{per}(W, \rho)$ will denote the $T$-algebra corresponding to the pointed flow (cls $\left.\left\{x_{0} \cdot t \mid t \in T\right\}, x_{0}\right) \subset\left(W_{\rho}, T\right)$. By (3.9) per $(W, \rho)$ is an almost periodic extension of $\mathscr{A}$.

(3.11) Proposition.

$$
z \circ \sigma \in Z_{\sigma}(\mathscr{A}, C / S)
$$

and

$z \circ \sigma+\sigma \in Z(\mathscr{A}, A / S)$ $(z \in Z(B / S, C / S))$.

(2) $\operatorname{per}(W, z \circ \sigma)=\operatorname{per}(\mathscr{S}, z \circ \sigma+\sigma) \quad(z \in Z(B / S, C / S))$.

(3) $\mathrm{g}\left(\operatorname{per}\left(\mathscr{S}, z^{\circ} \sigma+\sigma\right)\right)=\{a \in A \mid z(a B / S)=a C / S\}$.

(4) The maps $z \rightarrow z \circ \sigma: Z(B / S, C / S) \rightarrow Z_{\sigma}(\mathscr{A}, C / S)$ and $z \rightarrow z \circ \sigma+\sigma$ : $Z(B / S, C / S) \rightarrow Z(\mathscr{A}, A / S)$ are injective.

Proof. (1) This follows immediately from the definitions of the various terms involved.

(2) Since $(z \circ \sigma+\sigma)(\bar{x}, t)^{-1} x t=\sigma(\bar{x}, t)^{-1}\left(z \circ \sigma(x, t)^{-1} x t\right)=\left(z \circ \sigma(x, t)^{-1} x\right) \circ t$, the actions of $T$ on $|\mathscr{P}|$ used to determine $\operatorname{per}(W, z \circ \sigma)$ and $\operatorname{per}(\mathscr{P}, z \circ \sigma+\sigma)$ are the same. 
(3) Let $\quad \rho=z \circ \sigma+\sigma$. Then $\quad \delta \rho(x)=\rho(e, x)=z(\sigma(e, x))+\sigma(e, x)=$ $z(\delta \sigma(x))+\delta \sigma(x)(x \in \beta T)$. Then $g(\operatorname{per}(\mathscr{S}, \rho))=\{a \in A \mid \delta \rho(a)=a C / S\}$ [2]. Thus, if we write, $a=c+b$ with $c \in C, b \in B$ and recall that $\delta \sigma(c)=0$ and $\delta \sigma(b)=b S$, then $\mathfrak{g}(\operatorname{per}(\mathscr{I}, \rho))=\{a \in A \mid z(a B / S)=a C / S\}$.

(4) Let $z_{1}, z_{2} \in Z(B / S, C / S)$ and suppose $z_{1} \circ \sigma=z_{2} \circ \sigma$ or $z_{1} \circ \sigma+\sigma=z_{2} \circ \sigma+\sigma$. Then, in either case, $z_{1} \circ \sigma=z_{2} \circ \sigma$, whence $z_{1} \circ \delta \sigma=z_{2} \circ \delta \sigma$. By (3.7) above and (3.8.4) of [2], $\delta \sigma(A)=B / S$ and this implies that $z_{1}=z_{2}$. The proof is completed.

(3.12) Remarks. (1) Statement (3) above shows that the group of the flow $\operatorname{per}(\mathscr{P}, z \circ \sigma+\sigma)$ when projected onto $A / S$ is just the subgroup of $A / S$ determined by the cocycle $z$. (See proposition 2.4.) Thus all the elements of $\mathscr{C}^{\perp}$ may be realized by perturbing $\mathscr{S}$ by cocycles of the form $z \circ \sigma+\sigma$ or by perturbing $W$ by $\sigma$-cocycles of the form $z \circ \sigma(z \in Z(B / S, C / S))$.

(2) In their work on $\sigma$-extensions [4] Keynes and Newton ask what amounts in this context to the question: when is $|\operatorname{per}(W, \rho)|=W$ for $\rho \in Z_{\sigma}(\mathscr{A}, C / S)$. That this is the case when $\rho=z \circ \sigma$ for some $z \in Z(B / S, C / S)$ can be seen by examining the flow induced on $|\mathscr{P}|$ by the cocycle $\eta=z \circ \sigma+\sigma$. Then $x_{0} \cdot c=\delta \eta(x)^{-1} x_{0} c=x_{0} c(c \in C)$, since $C=\operatorname{ker} \delta \sigma$. Thus, in this case, $\pi^{-1}\left(\bar{x}_{0}\right) \subset|\operatorname{per}(W, \rho)|$, whence $|\operatorname{per}(W, \rho)|=W$. (Here $\pi: W \rightarrow|\mathscr{A}|$ is the canonical map.)

\section{The cohomology $H^{2}(L, J)$}

This section contains some more results from the theory of the cohomology of groups which will be used later. Proofs will be omitted or merely indicated. The data are those of $\S 2$ with the additional assumption that $J$ is abelian.

(4.1) Definition. A continuous function $f: L \times L \rightarrow J$ is a 2-cocycle on $L$ to $J$ if $x \cdot f(y, z)+f(x, y+z)=f(x, y)+f(x+y, z)$; and it is a 2-coboundary on $L$ to $J$ if there is a continuous function $g: L \rightarrow J$ with $f(x, y)=x \cdot g(y)-g(x+y)+g(x)(x, y, z \in L)$. (All functions involved are also assumed to be normalized, i.e. $f(x, 0)=f(0, y)=0$ and $g(0)=0(x, y \in L)$.)

Then the set of all 2-cocycles on $L$ to $J, Z^{2}(L, J)$ is an abelian group and the set of 2-coboundaries $B^{2}(L, J)$ a subgroup thereof. The quotient group $H^{2}(K, J)=$ $Z^{2}(K, J) / B^{2}(K, J)$ is the second cohomology group of $K$ with coefficients in $J$. (Again the reader is cautioned that here the functions considered are continuous whereas in the algebraic theory of the cohomology of groups there is no topology involved. Of course when $L$ is finite the two notions coincide.)

(4.2) Proposition. Let $f \in Z^{2}(L, J)$. Then the map $(x, y) \rightarrow x \oplus y=f(\pi(x), \pi(y))+$ $x+y: K \times K \rightarrow K$ defines a group structure on $K$ such that:

(1) $(K, \oplus)$ is a compact topological group.

(2) $x \oplus y=x+y, y \oplus x=y+x$, and $y \oplus x \ominus y=y+x-y(x \in J, y \in K)$.

(3) $x \oplus y-(x+y) \in J(x, y \in L)$.

Conversely, if $\oplus$ is a group structure on $K$ satisfying (1), (2) and (3), then the map $(x, y) \rightarrow x \oplus y-(x+y): L \times L \rightarrow J$ defines a 2-cocycle on $L$ to $J$.

(Here $\pi: K \rightarrow L$ is the map such that $\pi(a)=a L$, where $a=a J+a L(a \in K)$.)

The group structure determined by the 2 -cocycle $f$ will be denoted $(K, f)$. 
(4.3) Proposition. Let, $f, h \in Z^{2}(L, J)$. Then $f$ and $h$ are cohomologous (i.e. $\left.f-h \in B^{2}(L, J)\right)$ if and only if there exists an isomorphism $\phi$ of $(K, f)$ onto $(K, h)$ such that $\phi(x)=x(x \in J)$ and $\pi(\phi(x))=\pi(x)(x \in K)$.

Proof. The relation between the isomorphism $\phi$ and the function $g$ required in definition 4.1 is given by $\phi(x)=g(x)+x(x \in L)$.

5. The dynamical interpretation of $H^{2}(B / S, C / S)$

The situation is again that of $\S 3$ with the additional assumption that $C / S$ is abelian.

Let $f \in Z^{2}(B / S, C / S)$. Then, as in $\S 4$, a new topological group $(A / S, f)$ is produced. A natural question to ask is whether one can define an action of $(A / S, f)$ on $|\mathscr{P}|$ with quotient $|\mathscr{A}|$ such that the group of the resultant bundle can be reduced to $C / S$.

It turns out that this can be done (see 5.3). However, the resulting action need not commute with the action of $T$ on $|\mathscr{P}|$. Consequently, one seeks to perturb the latter in such a way as to obtain again a bitransformation group.

Perturbation suggests cocycles and an investigation shows that the relevant ones are the so called $B$-cocycles, $Z_{B}(\mathscr{C}, C / S)$ on $\mathscr{C}$ to $C / S$ (see 5.4).

Finally, it is shown how a $B$-cocycle, $\rho$, on $\mathscr{C}$ to $C / S$ determines a 2-cocycle on $B / S$ to $C / S$ and how the new group structure on $A / S$ is related to the group of the flow $\operatorname{per}(\mathscr{P}, \rho)$.

(5.1) Notation. For the remainder of this section $f$ will denote a fixed element of $Z^{2}(B / S, C / S)$,

and

$$
a_{1} \oplus a_{2}=f\left(\delta \sigma\left(a_{1}\right), \delta \sigma\left(a_{2}\right)\right)+a_{1}+a_{2},
$$

$a * x=(f(\delta \sigma(a), \delta \sigma(x))+a) x=f(\delta \sigma(a), \delta \sigma(x))(a x)\left(a_{1}, a_{2}, a \in A / S, x \in|\mathscr{S}|\right)$.

(Recall that $\delta \sigma$ induces the canonical map on $A / S$ to $B / S$.)

(5.2) LEMMA. $a * x=(a \oplus \alpha) y$ where $a, \alpha \in A / S, y \in W$ and $x=\alpha y$.

Proof. By (3.6), $\delta \sigma(y)=0$. Hence $\delta \sigma(x)=\delta \sigma(\alpha y)=\delta \sigma(\alpha)+\delta \sigma(y)=\delta \sigma(\alpha)$ and

$$
\begin{aligned}
a * x & =(f(\delta \sigma(a), \delta \sigma(x))+a) x=(f(\delta \sigma(a), \delta \sigma(\alpha))+a)(\alpha y) \\
& =(f(\delta \sigma(a), \delta \sigma(\alpha))+a+\alpha) y=(a \oplus \alpha) y .
\end{aligned}
$$

(5.3) Proposition. The map $(a, x) \rightarrow a * x: A / S \times|\mathscr{P}| \rightarrow|\mathscr{S}|$ defines a free action of $A / S$ on $|\mathscr{S}|$ such that:

(1) $c * x=c x(c \in C / S, x \in|\mathscr{S}|) ;$ and

(2) $a * x=a x(a \in A / S, x \in W)$.

Proof. Let $a_{1}, a_{2} \in A / S, x \in|\mathscr{S}|$. Write $x=\alpha y$ with $\alpha \in A / S, y \in W$. Then by (5.2),

$$
\begin{aligned}
a_{1} *\left(a_{2} * x\right) & =a_{1} *\left(\left(a_{2} \oplus \alpha\right) y\right)=\left(a_{1} \oplus\left(a_{2} \oplus \alpha\right)\right) y \\
& =\left(\left(\dot{a}_{1} \oplus a_{2}\right) \oplus \alpha\right) y=\left(a_{1} \oplus A_{2}\right) * \alpha y=\left(a_{1} \oplus a_{2}\right) * x .
\end{aligned}
$$

Now let $a * x=x$. Then $(a \oplus \alpha) y=\alpha y$, whence $a \oplus \alpha=\alpha$ and $a=0$.

(1) $c * x=(c \oplus \alpha) y=(c+\alpha) y=c(\alpha y)=c x(c \in C / S)$, by (2) of (4.2).

(2) If $x \in W$, then $a * x=(a \oplus 0) x=a x(a \in A / S)$, by (5.2).

The next step is to produce an action of $T$ on $|\mathscr{P}|$ which commutes with the action of $(A / S, f)$ defined in (5.3). Since the group structure on $C / S$ induced by $f$ coincides 
with the original group structure and the action of $C / S$ on $|\mathscr{P}|$ is unchanged, it is natural to seek an action of $T$ such that the canonical map of $|\mathscr{S}|$ onto $|\mathscr{C}|$ will be a homomorphism. This is equivalent to finding a cocycle $\rho$ on $\mathscr{C}$ to $C / S$, i.e. an element of $Z(\mathscr{C}, C / S)$, and using it to perturb the original flow on $|\mathscr{S}|$.

It turns out that not all the elements of $Z(\mathscr{C}, C / S)$ arise in this way (i.e. by varying $f$ ), only the so-called $B$-cocycles. It is convenient for expository purposes to study these objects before studying $f$ further.

(5.4) Definition. Let $\rho \in Z^{1}(\mathscr{C}, C / S)$. Then $\rho$ is a $B$-cocycle on $\mathscr{C}$ to $C / S$ if $\delta \rho(a x)=$ $a \cdot \delta \rho(x)+\delta \rho(a)(a \in A, x \in \beta T$ with $\delta \sigma(x)=0)$. The set of $B$-cocycles on $\mathscr{C}$ to $C / S$ will be denoted by $Z_{B}(\mathscr{C}, C / S)$.

(5.5) LeMMA. Let $\rho \in Z_{B}(\mathscr{C}, C / S)$. Then $d \delta \rho\left(a_{1}+c_{1}, a_{2}+c_{2}\right)=d \delta \rho\left(a_{1}, a_{2}\right)=$ $d \delta \rho\left(c_{1}+a_{1}, c_{2}+a_{2}\right)\left(c_{1}, c_{2} \in C, a_{1}, a_{2} \in A\right)$.

Proof. The coboundary operator $d$ satisfies the relation:

$$
d \delta \rho\left(\alpha_{1}, \alpha_{2}\right)=\bar{\alpha}_{1} \cdot \delta \rho\left(\alpha_{2}\right)-\delta \rho\left(\alpha_{1}+\alpha_{2}\right)+\delta \rho\left(\alpha_{2}\right)\left(\alpha_{1}, \alpha_{2} \in A\right) .
$$

By setting $\alpha_{1}=c_{1}+a_{1}$ one obtains $d \delta \rho\left(c_{1}+a_{1}, \alpha_{2}\right)=\overline{c_{1}+a_{1}}$.

$$
\begin{aligned}
\delta \rho\left(\alpha_{2}\right) & -\delta \rho\left(c_{1}+a_{1}+\alpha_{2}\right)+\delta \rho\left(c_{1}+a_{1}\right) \\
= & \bar{a}_{1} \cdot \delta \rho\left(\alpha_{2}\right)-\left(\delta \rho\left(c_{1}\right)+\delta \rho\left(a_{1}+a_{2}\right)\right)+\delta \rho\left(c_{1}\right)+\delta \rho\left(a_{1}\right) \\
= & \bar{a}_{1} \cdot \delta \rho\left(\alpha_{2}\right)-\delta \rho\left(a_{1}+\alpha_{2}\right)+\delta \rho\left(\alpha_{2}\right)=d \delta \rho\left(a_{1}, \alpha_{2}\right)
\end{aligned}
$$

(since the inner automorphism of $C / S$ induced by $\bar{c}_{1}$ is the identity).

Now set $\alpha_{2}=c_{2}+a_{2}$. Then $d \delta \rho\left(a_{1}, \alpha_{2}\right)=\bar{a}_{1} \cdot \delta \rho\left(c_{2}+a_{2}\right)-\delta \rho\left(a_{1}+c_{2}+a_{2}\right)+$ $\delta \rho\left(a_{1}\right)$. By (5.4), $\delta \rho\left(a_{1}+c_{2}+a_{2}\right)=\delta \rho\left(a_{1} \cdot c_{2}+a_{1}+a_{2}\right)=\delta \rho\left(a_{1} \cdot c_{2}\right)+\delta \rho\left(a_{1}+a_{2}\right)=$ $\bar{a}_{1} \cdot \delta \rho\left(c_{2}\right)+\delta \rho\left(a_{1}+a_{2}\right)$, whence $d \delta \rho\left(a_{1}, \alpha_{2}\right)=\bar{a}_{1} \cdot\left(\delta \rho\left(c_{2}\right)+\delta \rho\left(a_{2}\right)\right)-\bar{a}_{1} \cdot \delta \rho\left(c_{2}\right)-$ $\delta \rho\left(a_{1}+a_{2}\right)+\delta \rho\left(a_{1}\right)=d \delta \rho\left(a_{1}, a_{2}\right)$. Thus $d \delta \rho\left(a_{1}, a_{2}\right)=d \delta \rho\left(c_{1}+a_{2}, c_{2}+a_{2}\right)$.

If one replaces $c_{1}$ by $a_{1} \cdot c_{1}$ and $c_{2}$ by $a_{2} \cdot c_{2}$, one obtains $d \delta \rho\left(a_{1}, a_{2}\right)=$ $d \delta \rho\left(a_{1}+c_{1}, a_{2}+c_{2}\right)\left(c_{1}, c_{2} \in C, a_{1}, a_{2} \in A\right)$.

(5.6) Remark. (1) Lemma 5.5 shows that $d \delta \rho$ induces a continuous function on $B / S \times B / S$ to $C / S$. Clearly $d(d \delta \rho)=0$ so that $d \delta \rho$ is a 2 -cocycle on $B / S$ to $C / S$. It is, however, in general not a 2-coboundary for $\delta \rho$ need not induce a function of $B / S$ to $C / S$.

(2) Since $d \delta \rho \in Z^{2}(B / S, C / S)$, it induces a group structure on $A / S$ and an action of $A / S$ on $|\mathscr{S}|$. These will be denoted by $a_{1} \otimes a_{2}$ and $a \odot x$ respectively $\left(a_{1}, a_{2}, a \in\right.$ $A / S$ and $x \in|\mathscr{S}|)$.

(5.7) LeMma. Let $\rho \in Z_{B}(\mathscr{C}, C / S)$. Then $d \delta \rho(\delta \sigma(a), \delta \sigma(x))+\delta \rho(a x)=\delta \rho(a)+$ $a \cdot \delta \rho(x)(a \in A, x \in M)$. (Notice that $d \delta \rho(\delta \sigma(a), \delta \sigma(x))$ is well defined by (5.5).)

Proof. Let $a=c+b, \delta \sigma(x)=\bar{\beta}$ with $c \in C, b, \beta \in B$. Then $d \delta \rho(\delta \sigma(a), \delta \sigma(x))=$ $d \delta \rho(b, \beta)=\bar{b} \cdot \delta \rho(\beta)-\delta \rho(b+\beta)+\delta \rho(b)$ and $x=\beta y$ with $\delta \sigma(y)=0$.

Then $\delta \rho(a x)=\delta \rho((a+\beta) y)=(\overline{a+\beta}) \cdot \delta \rho(y)+\delta \rho(a+\beta)$, by $(5.4)$, and $a \cdot \delta \rho(x)=$ $\bar{a} \cdot \delta \rho(\beta y)=\bar{a} \cdot(\bar{\beta} \cdot \delta \rho(y)+\delta \rho(\beta))=\overline{a+\beta} \cdot \delta \rho(y)+\bar{a} \cdot \delta \rho(\beta)$.

Hence

$$
\begin{aligned}
& d \delta \rho(\delta \sigma(a), \delta \sigma(x))-a \cdot \delta \rho(x)+\delta \rho(a x) \\
& \quad=\bar{b} \cdot \delta \rho(\beta)-\bar{a} \cdot \delta \rho(\beta)-\delta \rho(b+\beta)+\delta \rho(a+\beta)+\delta \rho(b) .
\end{aligned}
$$


Since $\bar{b}-\bar{a}=\bar{c}$ and $C / S$ is abelian, $\bar{b} \cdot \delta \rho(\beta)=\bar{a} \cdot \delta \rho(\beta)$. Also $\delta \rho(a+\beta)=$ $\delta \rho(c+b+\beta)=\delta \rho(c)+\delta \rho(b+\beta)$. Consequently, $d \delta \rho(\delta \sigma(a), \delta \sigma(x))-a \cdot \delta \rho(x)+$ $\delta \rho(a x)=\delta \rho(c)+\delta \rho(b)=\delta \rho(a)$. The proof is completed.

(5.8) LemmA. Let $\rho \in Z_{B}(\mathscr{C}, C / S)$ and $\Gamma: A \rightarrow(A / S, d \delta \rho)$ such that $\Gamma(a)=$ $-\delta \rho(a)+\bar{a}(a \in A)$. Then $\Gamma$ is a homomorphism such that $\Gamma(C) \subset C / S$ and $\operatorname{ker} \Gamma \subset C$. Moreover, $\Gamma$ is onto if and only if $\Gamma(C)=C / S$.

Proof. Since $\delta \rho(A) \subset C / S, \Gamma(C) \subset C / S$.

Let $a_{1}, a_{2} \in A$. Then

by $(5.7)$.

$$
\begin{aligned}
\Gamma\left(a_{1}\right) \otimes \Gamma\left(a_{2}\right) & =d \delta \rho\left(\delta \sigma\left(\Gamma\left(a_{1}\right)\right), \delta \sigma\left(\Gamma\left(a_{2}\right)\right)\right)+\Gamma\left(a_{1}\right)+\Gamma\left(a_{2}\right) \\
& =d \delta \rho\left(\delta \sigma\left(a_{1}\right), \delta \sigma\left(a_{2}\right)\right)-\delta \rho\left(a_{1}\right)+\bar{a}_{1}-\delta \rho\left(a_{2}\right)+\bar{a}_{2} \\
& =d \delta \rho\left(\delta \sigma\left(a_{1}\right), \delta \sigma\left(a_{2}\right)\right)-\delta \rho\left(a_{1}\right)-\bar{a}_{1} \cdot \delta \rho\left(a_{2}\right)+\bar{a}_{1}+\bar{a}_{2} \\
& =-\delta \rho\left(a_{1}+a_{2}\right)+\bar{a}_{1}+\bar{a}_{2}=\Gamma\left(a_{1}+a_{2}\right)
\end{aligned}
$$

Thus $\Gamma$ is a homomorphism and $\Gamma(a)=0$ if and only if $\delta \rho(a)=\bar{a}$. Since $\delta \rho(A) \subset$ $C / S$ this can only occur if $a \in C$.

Moreover, $\delta \rho(A) \subset C / S$ implies that if $\Gamma$ is onto then $\Gamma(C)=C / S$.

Finally, suppose $\Gamma(C)=C / S$ and let $a \in A$. Choose $c \in C$ with $\Gamma(c)=\delta \rho(a)$. Then $\Gamma(c+a)=\Gamma(c) \oplus \Gamma(a)=\delta \rho(a) \oplus \Gamma(a)=\delta \rho(a)+\Gamma(a)=\bar{a}$. The proof is completed.

(5.9) Proposition. Let $\rho \in Z_{B}(\mathscr{C}, C / S), N=\{a \in A \mid \delta \rho(a)=\bar{a}\}, \mathcal{N}=\operatorname{per}(\mathscr{S}, \rho)$, $\phi:|\mathcal{N}| \rightarrow|\mathscr{S}|$ such that $\phi(x \mid \mathcal{N})=\delta \rho(x)^{-1}(x \mid \mathscr{S})(x \in M)$, and $\Gamma: A \rightarrow A / S$ such that $\Gamma(a)=-\delta \rho(a)+\bar{a}$. Then $\phi((x \mid \mathcal{N}) t)=\phi(x) \cdot t=\rho(x, t)^{-1}((x \mid \mathscr{S}) t)(x \in M, t \in T)$ and $\phi(a x \mid \mathcal{N})=\Gamma(a) \odot \phi(x)(a \in A, x \in M)$.

Proof. The map $\phi$ is just the identification map $p \mid \mathcal{N} \rightarrow x_{0} \cdot p=\delta \rho(p)^{-1}(p \mid \mathscr{S})$ (see section 5 of [2]). Hence $\phi$ is a transformation group monomorphism of $(|\mathcal{N}|, T)$ into $\left(|\mathscr{S}|_{\rho}, T\right)$.

By (5.8), $N$ is a normal subgroup of $A$ and, by [2], $N$ is the group of the flow, $\mathcal{N}$. Hence $\phi(a(x \mid \mathcal{N}))=\phi(a x \mid \mathcal{N})=\delta \rho(a x)^{-1}(a x \mid \mathscr{S})=(-\delta \rho(a x)+\bar{a})(x \mid \mathscr{S})(a \in A$, $x \in M$ ).

On the other hand, $\Gamma(a) \odot \phi(x \mid \mathcal{N})=\Gamma(a) \odot\left(\delta \rho(x)^{-1} x \mid \mathscr{P}\right)=(d \delta \rho(\delta \sigma(\Gamma(a))$, $\left.\left.\delta \sigma\left(\delta \rho(x)^{-1} x \mid \mathscr{S}\right)\right)+\Gamma(a)-\delta \rho(x)\right)(x \mid \mathscr{S})$. Since $\Gamma(a)=-\delta \rho(a)+\bar{a}$ and $\delta \rho(a), \delta \rho(x) \epsilon$ $C / S, \quad \delta \sigma(\Gamma(a))=\delta \sigma(a) \quad$ and $\quad \delta \sigma\left(\delta \rho(x)^{-1} x\right)=\delta \sigma(x)$. Hence $\quad \Gamma(a) \odot \phi(x \mid \mathcal{N})=$ $(d \delta \rho(\delta \sigma(a), \delta \sigma(x))-\delta \rho(a)-\bar{a} \cdot \delta \rho(x)+\bar{a})(x \mid \mathscr{S})=(-\delta \rho(a x)+\bar{a})(x \mid \mathscr{S})$. Consequently, $\Gamma(a) \odot \phi(x \mid \mathcal{N})=\phi(a(x \mid \mathcal{N}))$.

(5.10) LemMA. Let $\rho \in Z_{B}(\mathscr{C}, C / S)$. Then $c \odot(x \cdot t)=(c \odot x) \cdot t(c \in C / S, x \in|\mathscr{S}|$, $t \in T)$.

Proof. This follows immediately from the definitions of the terms involved.

(5.11) Proposition. Let $\rho, \Gamma$, and $\phi$ be as in (5.9). Then

(1) The flow $\left(|\mathscr{S}|_{\rho}, T\right)$ commutes with the action of $(A / S, d \delta \rho)$ on $|\mathscr{S}|$.

(2) The pair $(\Gamma, \phi)$ is a bitransformation group monomorphism of $(A / N,|\mathcal{N}|, T)$ into $\left((A / S, d \delta \rho),|\mathscr{S}|_{\rho}, T\right)$.

(3) $\phi$ is onto if and only if $\Gamma$ is onto. 
Proof. (1) Let $U$ be the image of $\phi$, and let $a \in A, x \in|\mathcal{N}|$ and $t \in T$. Since $(a x) t=a(x t)$, $(\Gamma(a) \odot \phi(x)) \cdot t=\phi(a x) \cdot t=\phi((a x) t)=\phi(a(x t))=\Gamma(a) \odot \phi(x t)=\Gamma(a) \odot(\phi(x) \cdot t)$. Hence $(\Gamma(a) \odot y) \cdot t=\Gamma(a) \odot(y \cdot t)(a \in A, y \in U, t \in T)$.

Now $\quad(\Gamma(a) \odot y) \cdot t=((-\delta \rho(a)+\bar{a}) \odot y) \cdot t=((-\delta \rho(a) \otimes \bar{a}) \odot y) \cdot t=$ $\left(\delta \rho(a)^{-1} \odot(a \odot y)\right) \cdot t=\delta \rho(a)^{-1}((a \odot y) \cdot t)$, since $\delta \rho(a) \in C / S$.

On the other hand, $\Gamma(a) \odot(y \cdot t)=(-\delta \rho(a)+\bar{a}) \odot(y \cdot t)=\delta \rho(a)^{-1}(a \odot(y \cdot t))$ (as above).

Hence $(a \odot y) \cdot t=a \odot(y \cdot t)(a \in A, y \in U, t \in T)$.

Let $x \in|\mathscr{S}|$. Then, since $U$ covers $|\mathscr{A}|$, there exist $\alpha \in A, y \in U$ with $x=\alpha \odot y$. Then $(a \odot x) \cdot t=(a \odot(\alpha \odot y)) \cdot t=((a \otimes \alpha) \odot y) \cdot t=(a \otimes \alpha) \odot(y \cdot t)$

$$
=a \odot(\alpha \odot(y \cdot t))=a \odot((\alpha \odot y) \cdot t)=a \odot(x \cdot t) \text {. }
$$

(2) This is just (5.9).

(3) Let $\phi$ be onto. Since $\phi$ preserves fibres over $|\mathscr{A}|$, it maps $A / N$ onto $A / S$. But $\phi \mid A / N=\Gamma$.

On the other hand, if $\Gamma$ is onto, $\phi$ maps the fibre over the base point of $|\mathscr{A}|$ onto itself. Since $\phi$ is a $T$-homomorphism and $(|\mathscr{A}|, T)$ is minimal, $\phi$ is onto.

The following result from the algebraic theory of minimal flows will be useful (see 12.12 of [1]).

(5.12) Proposition. Let $(H, X, T)$ be a bitransformation group with $(X, T)$ minimal, $\mathscr{F}=\mathrm{al}\left(X / H, y_{0}\right), \mathscr{L}=\mathrm{al}\left(X, x_{0}\right), \Gamma$ the map from $F=\mathrm{g}(\mathscr{F})$ to $H$ such that $\Gamma(a)\left(x_{0}\right)=$ $x_{0} a(a \in F)$ and $\phi$ the map $p\left|\mathcal{N} \rightarrow x_{0} p:\right| \mathscr{L} \mid \rightarrow X(p \in M)$. Then the pair $(\Gamma, \phi)$ is a bitransformation group isomorphism of $(F / L,|\mathscr{L}|, T)$ onto $(H, X, T)$.

(5.13) Proposition. Let $\rho \in Z(\mathscr{C}, C / S)$ and $f \in Z^{2}(B / S, C / S)$ be such that $\left((A / S, f),|\mathscr{P}|_{\rho}, T\right)$ is a bitransformation group. Then $\rho \in Z_{B}(\mathscr{C}, C / S)$ and $f=d \delta \rho$.

Proof. Let $\quad \mathcal{N}=\operatorname{per}(\mathscr{S}, \rho), \quad N=\mathfrak{g}(\mathcal{N}), \quad X=\operatorname{cls}\left\{x_{0} \cdot t \mid t \in T\right\} \subset|\mathscr{S}| \quad$ and $\quad H=$ $\{a \in A / S \mid a * x \in X(x \in X)\}$. Then (5.12) is applicable and the pair $(\Gamma, \phi)$ becomes in this case $\phi(p \mid \mathcal{N})=x_{0} \cdot p=\delta \rho(p)^{-1} x_{0} p=\delta \rho(p)^{-1}(p \mid \mathscr{S}) \quad(p \in M)$ and $\Gamma(a) x_{0}=$ $\Gamma(a) * x_{0}=\Gamma(a)\left(x_{0}\right)=x_{0} \cdot a=\delta \rho(a)^{-1}\left(x_{0} a\right)=(-\delta \rho(a)+\bar{a}) x_{0}$. Hence, viewed as a map from $A$ into $(A / S, f), \Gamma(a)=-\delta \rho(a)+\bar{a}(a \in A)$.

Now let $\delta \sigma(x)=0$ and $a \in A$. Then $\phi(a x)=\delta \rho(a x)^{-1}(a x \mid \mathscr{S})=$ $(-\delta \rho(a x)+\bar{a})(x \mid \mathscr{S})$. Also $\phi(a x)=\Gamma(a) * \phi(x)=(f(\delta \sigma(\Gamma(a)), \delta \sigma(\phi(x))+\Gamma(a)) \phi(x)$. Since $\phi(x)=\delta \rho(x)^{-1}(x \mid \mathscr{S}), \delta \sigma(\phi(x))=\delta \sigma\left(\delta \rho(x)^{-1}\right)+\delta \sigma(x)=0$ (recall that $\delta \rho(x) \in$ $C / S$ and $C=\operatorname{ker} \delta \sigma)$. Thus $\phi(a x)=\Gamma(a) \phi(x)=(-\delta \rho(a)+\bar{a})\left(\delta \rho(x)^{-1} x \mid \mathscr{S}\right)=$ $(-\delta \rho(a)-a \cdot \delta \rho(x)+\bar{a})(x \mid \mathscr{S})$. Equating the two expressions for $\phi(a x)$, one obtains $\delta \rho(a x)=a \cdot \delta \rho(x)+\delta \rho(a)$; i.e. $\rho$ is a $B$-cocycle.

By (5.8) and the first part of this proof, $\Gamma\left(a_{1}\right) \oplus \Gamma\left(a_{2}\right)=\Gamma\left(a_{1}\right) \otimes \Gamma\left(a_{2}\right)\left(a_{1}, a_{2} \in A\right)$. Since $a_{i}=-\delta \rho\left(a_{i}\right)+\Gamma\left(a_{i}\right)$ and $\delta \rho\left(a_{i}\right) \in C / S(i=1,2), a_{1} \oplus a_{2}=a_{1} \otimes a_{2}$, whence $f\left(a_{1}, a_{2}\right)=d \delta \rho\left(a_{1}, a_{2}\right)\left(a_{1}, a_{2} \in A\right)$.

Proposition 5.11 shows that a $B$-cocycle $\rho$ on $\mathscr{C}$ to $C / S$ produces a group structure on $A / S$ and a flow $|\mathscr{P}|_{\rho}$ on $|\mathscr{S}|$ such that $\left((A / S, d \delta \rho),|\mathscr{P}|_{\rho}, T\right)$ is a bitransformation group. It would be desirable to know whether, given $f \in Z^{2}(B / \mathcal{S}, C / S)$, there is 
$\rho \in Z_{B}(\mathscr{C}, C / S)$ with $d \delta \rho=f$. The next few results make use of proposition (5.13) to give a partial answer to this question.

(5.14) Proposition. Let $T=\mathbb{Z}$ and $f \in Z^{2}(B / S, C / S)$. Then there exists $\rho \in$ $Z_{B}(\mathscr{C}, C / S)$ with $d \delta \rho=f$.

Proof. Let $t$ be the generator of $\mathbb{Z}$. By (5.13) it suffices to find a map $x \mapsto x \cdot t:|\mathscr{S}| \rightarrow$ $|\mathscr{S}|$ such that $a *(x \cdot t)=(a * x) \cdot t(a \in A / S, x \in|\mathscr{S}|)$.

To this end set $x \cdot t=f(\delta \sigma(x), \sigma(\bar{x}, t)) x t$. Then

$$
\begin{aligned}
a *(x \cdot t) & =(f(\delta \sigma(a), \delta \sigma(x t))+a+f(\delta \sigma(x), \sigma(\bar{x}, t))) x t \\
& =(f(\delta \sigma(a), \delta \sigma(x t))+a \cdot f(\delta \sigma(x), \sigma(\bar{x}, t))+a) x t,
\end{aligned}
$$

and

$(a * x) \cdot t=(f(\delta \sigma(a), \delta \sigma(x))+a) x \cdot t=(f(\delta \sigma(a x), \sigma(\bar{x}, t))+f(\delta \sigma(a), \delta \sigma(x))+a) x t$.

Since $a \cdot c=\delta \sigma(a) \cdot c, \delta \sigma(a x)=\delta \sigma(a)+\delta \sigma(x)$ and $\sigma(\bar{x}, t)=-\delta \sigma(x)+\delta \sigma(x t)$, the preceding equations and the fact that $f$ is a 2-cocycle imply that $(a * x) \cdot t=a *(x \cdot t)$.

(5.15) Proposition. Let $T$ be free, al $(\sigma)=\mathbb{R}$, and $f \in Z^{2}(B / S, C / S)$. Then there exists $\rho \in Z_{B}(\mathscr{C}, C / S)$ with $d \delta \rho=f$.

Proof. Since $\delta \sigma$ induces a homomorphism of $T$ into $B / S$ [2], the map $(t, c) \rightarrow \delta \sigma(t) \cdot c$ of $T \times C / S$ into $C / S$ defines an action of $T$ on $C / S$.

A straightforward computation shows that the map $\left(t_{1}, t_{2}\right) \rightarrow$ $f\left(\delta \sigma\left(t_{1}\right), \delta \sigma\left(t_{2}\right)\right): T \times T \rightarrow C / S$ is a 2-cocycle on $T$ to $C / S$ (see [5]).

Since $T$ is free, this map is a coboundary, i.e. there exists $w: T \rightarrow C / S$ with $f\left(\delta \sigma\left(t_{1}\right), \delta \sigma\left(t_{2}\right)\right)=d w\left(t_{1}, t_{2}\right)=\delta \sigma\left(t_{1}\right) \cdot w\left(t_{2}\right)-w\left(t_{1} t_{2}\right)+w\left(t_{1}\right)$ and $w(0)=0$ [5].

We may extend $w$ to $\beta T$ and, by continuity, obtain

$$
f(\delta \sigma(x), \delta \sigma(y))=\delta \sigma(x) \cdot w(y)-w(x y)+w(x) \quad(x, y \in \beta T) .
$$

Now set $\rho(x, t)=-w(x)+w(x t)=-w(x)+w(x)+\delta \sigma(x) \cdot w(t)-f(\delta \sigma(x), \delta \sigma(t))=$ $\delta \sigma(x) \cdot w(t)-f(\delta \sigma(x), \delta \sigma(t))$. This shows that $\rho$ is a cocycle on $\mathscr{C}$ to $C / S$ with $\delta \rho=w$.

If one sets $x=a$ and $\delta \sigma(y)=0$ in $(*)$, one obtains $0=\delta \sigma(a) \cdot w(y)-w(a y)+w(a)$; i.e. $\rho$ is a $B$-cocycle.

Finally, $\delta \sigma(b)=\vec{b}(b \in B)$ implies that $d \delta \rho=d w=f$. The proof is completed.

(5.16) Proposition. Let $f \in B^{2}(B / S, C / S)$. Then there exists $\rho \in Z_{B}(\mathscr{C}, C / S)$ with $d \delta \rho=f$. Moreover, $\rho \in B^{1}(\mathscr{C}, C / S)$ and $(A / S,|\mathscr{S}|, T) \cong\left((A / S, f),|\mathscr{S}|_{\rho}, T\right)$.

Proof. Let $g: B / S \rightarrow C / S$ be such that $g(0)=0$ and $d g=f$. Set $w(x)=g(\delta \sigma(x))$ $(x \in \beta T)$. Then $w$ induces a continuous map from $|\mathscr{C}| \rightarrow C / S$. Hence the equation $\rho(x, t)=w(x)^{-1} w(x t)(x \in \beta T)$ defines a coboundary $\rho$ on $\mathscr{C}$ to $C / S$ with $\delta \rho=w$.

Let $\delta \sigma(x)=0$ and $a \in A$. Then $\delta \rho(a x)=w(a x)=g(\delta \sigma(a x))=g(\delta \sigma(a)+\delta \sigma(x))=$ $g(\delta \sigma(a)) \quad$ and $\quad w(x)=g(\delta \sigma(x))=g(0)=0$. Hence $\quad \delta \rho(a x)=g(\delta \sigma(a))=$ $a \cdot \delta \rho(x)+\delta \rho(a)$ and so $\rho \in Z_{B}(B / S, C / S)$.

Moreover, $d \delta \rho\left(b_{1}, b_{2}\right)=d w\left(b_{1}, b_{2}\right)=b_{1} \cdot w\left(b_{2}\right)-w\left(b_{1}+b_{2}\right)+w\left(b_{1}\right)=b_{1} \cdot g\left(b_{2}\right)-$ $g\left(b_{1}+b_{2}\right)+g\left(b_{1}\right)=d g\left(b_{1}, b_{2}\right)=f\left(b_{1}, b_{2}\right)\left(b_{1}, b_{2} \in B / S\right)$. 
Finally, in this case the map $\Gamma$ with $\Gamma(a)=-\delta \rho(a)+\tilde{a}$ reduces to the canonical map of $C$ onto $C / S$. Hence the group $N$ of the flow $\mathcal{N}=\operatorname{per}(\mathscr{S}, \rho)$ is just $S$ by (5.9), and (5.16) follows from (5.8) and (5.11).

I should now like to relate $\S 3$ to the present section. To this end the following lemma shows how the set of $\sigma$-cocycles on $\mathscr{A}$ to $C / S$ may be included in the set of $B$-cocycles on $\mathscr{C}$ to $C / S$.

(5.17) LemmA. Let $\Phi(\gamma) \cdot(x, t)=\delta \sigma(x) \cdot \gamma(\bar{x}, t)\left(x \in|\mathscr{C}|, t \in T, \gamma \in Z_{\sigma}(\mathscr{A}, C / S)\right)$. Then $\Phi$ is a monomorphism of $Z_{\sigma}(\mathscr{A}, C / S)$ into $Z_{B}(\mathscr{C}, C / S)$.

Proof. That $\Phi$ is a monomorphism of $Z_{\sigma}(\mathscr{A}, C / S)$ into $Z(\mathscr{C}, C / S)$ follows immediately from the definitions involved.

Now $\delta \Phi(\gamma)(a x)=\Phi(\gamma)(e, a x)=\delta \sigma(e) \cdot \gamma(e, a x)=\gamma(e, a x)=\gamma(e, a)+\sigma(e, a) \cdot \gamma(a, c)$; $\delta \Phi(\gamma)(a)=\gamma(e, a)$ and $a \cdot \delta \Phi(\gamma)(x)=a \cdot \gamma(e, x)$. Since $\gamma$ induces a function on $|\mathscr{A}|$, $\gamma(a, x)=\gamma(e, x)$. Moreover, since $C / S$ is abelian, $a \cdot c=\delta \sigma(a) \cdot c=\sigma(e, a) \cdot c(a \in A$, $c \in C / S)$. Consequently, $\Phi(\gamma) \in Z_{B}(B / S, C / S)$.

(5.18) Proposition. Let $\rho \in Z_{B}(\mathscr{C}, C / S)$. Then the following statements are pairwise equivalent.

(1) $\rho \in \operatorname{im} \Phi$.

(2) $\left(A / S,|\mathscr{S}|_{\rho}, T\right)$ is a bitransformation group.

(3) $d \delta \rho=0$.

Proof. (1) implies (2). Let $\rho(x, t)=\delta \sigma(x) \cdot \gamma(\bar{x}, t)$ with $\gamma \in Z_{\sigma}(\mathscr{A}, C / S)$, and let $a \in A / S, x \in|\mathscr{S}|$. Then $a(x \cdot t)=a\left(\rho(x, t)^{-1} x t\right)=(a-\rho(x, t))(x t)=(-a \cdot \rho(x, t))(a x t)$ and $(a x) \cdot t=\rho(a x, t)^{-1}(a x t)=(-\rho(a x, t))(a x t)$.

Now

$$
\begin{aligned}
\rho(a x, t) & =\delta \sigma(a x) \cdot \gamma(\overline{a x}, t)=(\delta \sigma(a)+\delta \sigma(x)) \cdot \gamma(\bar{x}, t) \\
& =\delta \sigma(a) \cdot(\delta \sigma(x) \cdot \gamma(\bar{x}, t)=a \cdot \rho(x, t) .
\end{aligned}
$$

(2) implies (3) by (5.13) with $f \equiv 0$.

(3) implies (1). Set $\gamma(x, t)=\delta \sigma(x)^{-1} \cdot \rho(x, t)=\delta \sigma(x)^{-1} \cdot(-\delta \rho(x)+\delta \rho(x t))$. Let $a \in A$. Then

$$
\begin{aligned}
\gamma(a x, t) & =\delta \sigma(a x)^{-1}:(-\delta \rho(a x)+\delta \rho(a x t)) \\
& =\delta \sigma(a x)^{-1} \cdot(-a \cdot \delta \rho(x)-\delta \rho(a)+\delta \rho(a)+a \cdot \delta \rho(x t)) \\
& =(-\delta \sigma(x)-\delta \sigma(a)) \cdot a \cdot \rho(x, t)=\delta \sigma(x)^{-1} \cdot \rho(x, t)=\gamma(x, t) .
\end{aligned}
$$

Thus $\gamma$ defines a continuous function from $|\mathscr{A}| \times T$ to $C / S$. The verification that $\gamma$ is a $\sigma$-cocycle is straightforward. The proof is completed.

(5.19) Proposition. Let $Z_{B}(\mathscr{S})=\left\{\eta \in Z_{B}(\mathscr{C}, C / S) \mid \operatorname{per}(\mathscr{S}, \eta)=\mathscr{S}\right\}$. Then $d \delta \eta \in$ $B^{2}(B / S, C / S)\left(\eta \in Z_{B}(\mathscr{S})\right)$, and the map $\Delta$ such that $\Delta(\eta)=d \delta \eta$ is a homomorphism of $Z_{B}(\mathscr{S})$ onto $B^{2}(B / S, C / S)$ with $\operatorname{ker} \Delta=\operatorname{im} \Phi$.

Proof. Since the group of $\operatorname{per}(\mathscr{S}, \eta)$ is $S, A / S \cong(A / S, d \delta \eta)(5.11)$, whence $d \delta \eta \in$ $B^{2}(B / S, C / S)(4.3)$. (The isomorphism in (5.11) is the identity on $C / S$.) 
Let $f \in B^{2}(B / S, C / S)$. Then there exists $\eta \in Z_{B}(\mathscr{C}, C / S)$ with $d \delta \eta=f$ and $\eta \in$ $B(\mathscr{C}, C / S)(5.16)$. Thus $\delta \eta(c)=0(c \in C)$, whence the group of $\operatorname{per}(\mathscr{S}, \eta)=$ $\{a \in A \mid \delta \eta(a)=\bar{a}\}=S$. Consequently, $\mathscr{S}=\operatorname{per}(\mathscr{P}, \eta)$ and $\Delta$ is onto.

The map $\Delta$ is clearly a homomorphism and its kernel is the image of $\Phi$ by $(5.18)$.

The proof of (5.19) together with (5.14) implies:

(5.20) Proposition. Let $T=\mathbb{Z}$. Then the map $\rho \mapsto d \delta \rho: Z_{B}(\mathscr{C}, C / S) \rightarrow$ $Z^{2}(B / S, C / S)$ induces an isomorphism of $Z_{B}(\mathscr{C}, C / S) / Z_{B}(\mathscr{S})$ onto $H^{2}(B / S, C / S)$.

In (3.11) it was shown that the map $z \mapsto z \circ \sigma: Z(B / S, C / S) \rightarrow Z_{\sigma}(\mathscr{A}, C / S)$ is injective. Combining this map with the embedding of $Z_{\sigma}(\mathscr{A}, C / S)$ into $Z_{B}(\mathscr{C}, C / S)$ (5.17), one obtains an embedding $\Psi$ of $Z(B / S, C / S)$ in $Z_{B}(\mathscr{C}, C / S)$. The next result characterizes the image of $\Psi$.

(5.21) Proposition. Let $P(\rho)=\delta \rho \circ \sigma \quad\left(\rho \in Z_{B}(\mathscr{C}, C / S)\right)$. Then $P$ induces $a$ homomorphism of $\operatorname{im} \Phi \cap B(\mathscr{C}, C / S)$ onto im $\Psi$ with $P^{2}=P$ and $\operatorname{ker} P=$ $\Phi\left(B_{\sigma}(\mathscr{A}, C / S)\right)$.

Proof. Let $\rho \in \operatorname{im} \Phi \cap B(\mathscr{C}, C / S)$. Then $\delta \rho(c)=0(c \in C)$ and so $\delta \rho$ induces a map of $B / S$ into $C / S$. By (3) of (5.18), $\delta \rho \in Z(B / S, C / S)$, whence $P(\rho) \in \operatorname{im} \Psi$. Since $\delta\left(\rho_{1}+\rho_{2}\right)=\delta \rho_{1}+\delta \rho_{2}, P$ is a homomorphism of im $\Phi \cap B(\mathscr{C}, C / S)$ into im $\Psi$.

Let $\eta \in \operatorname{im} \Psi$. Then $\eta(x, t)=\delta \sigma(x) \cdot g(\sigma(\bar{x}, t)) \quad(x \in|\mathscr{C}|, t \in T)$ where $g \in$ $Z(B / S, C / S)$. Then $\eta \in \operatorname{im} \Phi$ and $\delta \eta=g \circ \delta \sigma$. Since $\delta \sigma(c)=0(c \in C), \eta \in B(\mathscr{C}, C / S)$. Consequently, $\eta \in \operatorname{im} \Phi \cap B(\mathscr{C}, C / S)$.

Now $P(\eta)=\delta \eta \circ \sigma$, i.e. $P(\eta)(x, t)=\delta \eta(\sigma(x, t))=g(\delta \sigma(\sigma(x, t))=g(\sigma(x, t))=$ $\eta(x, t)(\delta \sigma(b)=b(b \in B / S))$. Thus $P(\eta)=\eta$.

This shows that $P$ is onto and that $P^{2}=P$.

Let $\rho(x, t)=\delta \sigma(x) \cdot \gamma(\bar{x}, t)$ with $\gamma \in B_{\sigma}(\mathscr{A}, C / S)$ then $P(\rho)(x, t)=\delta \rho(\sigma(x, t))=$ $\delta \gamma(\sigma(x, t))=0$.

On the other hand, let $\rho(x, t)=\delta \sigma(x) \cdot \gamma(\bar{x}, t)$ be in $B(\mathscr{C}, C / S)$ with $P(\rho)=0$. Then $\delta \rho=\delta \gamma$ implies $\delta \gamma(c)=0(c \in C)$ (since $\rho \in B(\mathscr{C}, C / S)$ ) and $\delta \gamma(b)=0(b \in B)$ (since image of $\sigma$ is $B / S$ and $\delta \gamma(\sigma(x, t))=\delta \rho(\sigma(x, t))=P(\rho)(x, t)=0)$. Hence $\gamma \in$ $B(\mathscr{C}, C / S)$. The proof is completed.

\section{Concluding remarks}

First I should like to discuss a simple example in order to illustrate some of the ideas in $\S \S 3$ and 5 .

Let us begin with the minimal flow $(|\mathscr{A}|, \alpha)$ where $|\mathscr{A}|$ is the set of reals $\bmod 1$ and $\alpha$ denotes the map $y \mapsto y+\alpha:|\mathscr{A}| \rightarrow|\mathscr{A}|$ with $\alpha$ irrational.

Let $\sigma$ be the cocycle on $\mathscr{A}$ to $\mathbb{Z}_{2}$ given by the constant map $y \rightarrow 1:|\mathscr{A}| \rightarrow \mathbb{Z}_{2}$. Then the flow $\mathscr{C}=\operatorname{ext}(\mathscr{A}, \sigma)$ is given by the $\operatorname{map}(a, y) \rightarrow(a+1, y+\alpha): \mathbb{Z}_{2} \times|\mathscr{A}| \rightarrow \mathbb{Z}_{2} \times|\mathscr{A}|$.

The flow $\mathscr{B}$ will be a $\sigma$-extension of $\mathscr{A}$. To construct it let $J$ denote the underlying topological group of $|\mathscr{A}|$ and $\phi$ a continuous map from $|\mathscr{A}|$ to $J$ such that the flow $(J \times|\mathscr{A}|, \gamma)$ is minimal, where $(k, y) \gamma=(\phi(y)-k, y+\alpha)(k \in J, y \in|\mathscr{A}|)$. (Many such $\phi$ exist, see [4].) 
Now $J$ acts on $J \times|\mathscr{A}|$ in the obvious way and $\mathbb{Z}_{2}$ acts on $J$ via the map $(a, k) \mapsto(-1)^{a} k: \mathbb{Z}_{2} \times J \rightarrow J$. Then it is easy to see that $(k(r, y)) \gamma=(\sigma(y) k)(r, y) \gamma$ $(k, r \in J, y \in|\mathscr{A}|)$; i.e. $\mathscr{B}$ is what Keynes and Newton call a $\sigma$-extension of $\mathscr{A}$.

The flow $\mathscr{S}=\mathscr{C} \vee \mathscr{B}$ is the subset of the cartesian product $|\mathscr{C}| \times|\mathscr{B}|$ given by the orbit closure of the point $(0,0,0,0)$ under the map

$$
(a, y, k, z) \mapsto(a+1, y+\alpha, \phi(z)-k, z+\alpha) .
$$

$\mathscr{P}$ may be identified with the flow $(|\mathscr{P}|, \eta)$, where $|\mathscr{P}|=\mathbb{Z}_{2} \times|\mathscr{A}| \times J$ and $(a, y, k) \eta=(a+1, y+\alpha, \phi(y)-k)$.

Let me now identify the various other objects which occur in $\S \S 3$ and 5 . Thus $C / S=J, B / S=\mathbb{Z}_{2}$ and $\boldsymbol{A} / \boldsymbol{S}=J \cdot \mathbb{Z}_{2}$, where $(k, a)(r, b)=\left(k+(-1)^{a} r, a+b\right)$. Then $A / S$ acts on $|\mathscr{S}|$ via the relation $(k, a)(b, y, r)=\left(a+b, y,(-1)^{a+b} k+r\right)$, and with this action $(A / S,|\mathscr{P}|, \eta)$ becomes a bitransformation group.

Then $C / S \cong J \cong\{(k, 0) \mid k \in J\} \subset A / S$ and $B / S \cong \mathbb{Z}_{2} \cong\left\{(0, a) \mid a \in \mathbb{Z}_{2}\right\} \subset A / S$. The flow $\operatorname{per}(\mathscr{S}, \sigma)$ is obtained from $\mathscr{S}$ by taking the orbit closure of the point $(0,0,0)$ under the flow induced by the map $(a, y, k) \mapsto \sigma(y)^{-1}((a, y, k) \eta)=$ $(0,1)(a+1, y+\alpha, \phi(y)-k)=(a, y+\alpha, \phi(y)-k)$. Thus

$$
W=\{(0, y, k)|y \in| \mathscr{A} \mid, k \in J\} \subset|\mathscr{S}| \text { and } \operatorname{per}(\mathscr{P}, \sigma)=\mathscr{B} \text {. }
$$

Let $x_{0}=(0,0,0) \in|\mathscr{S}|$. Since $\sigma(x, \eta)=(0,1)(x \in|\mathscr{S}|)$, the cocycle equation shows that $\delta \sigma\left(x_{0} \eta^{k}\right)=(0,0)$ or $(0,1)$ depending upon whether $k$ is even or odd. Hence $\delta \sigma(a, y, k)=(0, a)\left(a \in \mathbb{Z}_{2}, y \in|\mathscr{A}|, k \in J\right)$.

In this example $Z^{1}(B / S, C / S)=Z^{1}\left(\mathbb{Z}_{2}, J\right)=B^{1}\left(\mathbb{Z}_{2}, J\right)$ where $k \in J$ is identified with the cocycle $\rho_{k}$ on $\mathbb{Z}_{2}$ to $J$ such that $\rho_{k}(a)=k^{a}\left(a \in \mathbb{Z}_{2}\right)$. The corresponding flows on $W$ are given by the maps $\eta_{k}: W \rightarrow W(k \in J)$ where $(r, y) \eta_{k}=(\phi(y)-r-k, y+\alpha)$ $\left(r, k \in J, y \in|\mathscr{A}|\right.$ ), and the subgroups of $A / S$ by $\{(0,0),(k, 1)\}$ (consider $\eta_{k}{ }^{\circ} \sigma$ as in (3.11)).

The second cohomology group $H^{2}(B / S, C / S)$ is just $H^{2}(\mathbb{Z}, J)=\mathbb{Z}_{2}$ (see (7.1) of [5]) and a non-trivial 2-cocycle is given by the function

$$
f(a, b)= \begin{cases}\frac{1}{2} & \text { if } a=1=b \\ 0 & \text { otherwise }\end{cases}
$$

The new group structure on $J \cdot \mathbb{Z}_{2}$ is given by $(k, a) \oplus(r, b)=$ $\left(f(a, b)+k+(-1)^{a} r, a+b\right)$ and the new action of $J \cdot \mathbb{Z}_{2}$ on $|\mathscr{P}|$ by $(k, a) *(b, y, r)=$ $((f(a, b), 0)(k, a))(b, y, r)$. Thus $(k, a) \oplus(r, b)=(k, a)(r, b)$ and $(k, a) *(b, y, r)=$ $(k, a)(b, y, r)$, unless $a=1=b$ in which case $(k, 1) \oplus(r, 1)=\left(\frac{1}{2}+k-r, 0\right)$ and $(k, 1) *(1, y, r)=\left(\left(\frac{1}{2}, 0\right)(k, 1)\right)(1, y, r)=\left(k+\frac{1}{2}, 1\right)(1, y, r)=\left(0, y, k+\frac{1}{2}+r\right)$.

The new flow on $|\mathscr{S}|$ determined by the $B$-cocycle $\rho$ on $\mathscr{C}$ to $C / S$ with $d \delta \rho=f$ is given by the map $x \gamma=f(\delta \sigma(x), \sigma(x, \eta))(x \eta)(x \in|\mathscr{F}|)$. Thus, if $x=(b, y, r), \sigma(x, \eta)=$ $1, \delta \sigma(x)=b$ and $(b, y, r) \gamma=(f(b, 1), 0)(b, y, r) \eta=(f(b, 1), 0)(b+1, y+\alpha, \phi(y)-r)$. Thus $(0, y, r) \gamma=(0, y, r) \eta$ and $(1, y, r) \gamma=\left(\frac{1}{2}, 0\right)(1, y, r) \eta=\left(\frac{1}{2}, 0\right)(0, y+\alpha, \phi(y)-r)=$ $\left(0, y+\alpha, \phi(y)-r+\frac{1}{2}\right)\left(a, b \in \mathbb{Z}_{2}, r \in J, y \in|\mathscr{A}|\right)$.

I should like to conclude with some remarks, questions, and suggestions for further study. 
(6.1) Remarks. (1) The theory given in $\S 5$ is deficient in one point: it says little about the map $\rho \mapsto d \delta \rho: Z_{B}(\mathscr{C}, C / S) \rightarrow Z^{2}(B / S, C / S)$ for general groups $T$.

(2) The assumption that $C / S$ be abelian is necessary in order to apply the standard results of the cohomology theory of groups. However, in many of the situations arising in topological dynamics $C / S$ need not be abelian. This might be circumvented by emphasizing $Z_{B}(\mathscr{C}, C / S)$ rather than $H^{2}(B / S, C / S)$.

(3) In this paper $\mathscr{S}$ was assumed to be an almost periodic extension of $\mathscr{A}$. Can the results be extended to distal extensions?

(4) The question as to when there exists a cocycle $\sigma$ on $\mathscr{A}$ to $A / S$ with $\mathscr{C}=$ ext $(\mathscr{A}, \sigma), \mathscr{B}=\operatorname{per}(\mathscr{S}, \sigma)$ and $\mathscr{S}=\mathscr{C} \vee \mathscr{B}$ is an interesting one. However, starting with a flow $\mathscr{A}$ and a cocycle $\gamma$ on $\mathscr{A}$ to a compact group $K$ with $\delta \gamma(A)=K$, one can always produce $\mathscr{C}, \mathscr{B}, \mathscr{S}$ and $\sigma$ satisfying the preceding conditions: set $C=\operatorname{ker} \delta \gamma$ and choose any $\tau$-closed subgroup $B$ with $C B=A$. Then $S=C \cap B, \mathscr{C}=\mathscr{A}(C) \cap \mathscr{A}^{*}$, $\mathscr{B}=\mathscr{A}(B) \cap \mathscr{A}^{*}, \mathscr{S}=\mathscr{C} \vee \mathscr{B}$ and $\sigma(x, t)=\pi \delta \gamma^{-1}(\gamma(x, t))(x \in|\mathscr{A}|, t \in T)$ are the required objects. (Here $\pi$ is the isomorphism of $A / C$ onto $B / S$ induced by the canonical map of $A / S=C / S \cdot B / S$ onto $B / S$.) Of course, one may choose $B=A$ whereupon everything collapses. However, the set of possible choices for $B$ should give some insight into the flow $\mathscr{A}$ and the group $C$.

This research was supported by NSF Grant no. 7800403 AO2.

\section{REFERENCES}

[1] R. Ellis. Lectures on Topological Dynamics. Benjamin: New York, 1969.

[2] R. Ellis. Cocycles in topological dynamics. Topology 17 (1978), 111-130.

[3] R. Ellis. Cohomology of Flows. Lecture Notes in Math. no. 668. Springer: New York, 1978.

[4] H. Keynes \& D. Newton. Minimal $(G, \tau)$-extensions. Pacific Jour. of Math. 27 (1978), 145-163.

[5] S. MacLane. Homology. Academic Press: New York, 1963. 